\title{
Evidence for attractive pair interaction in diffusive gold films deduced from studies of the superconducting proximity effect with aluminum
}

\author{
M. Wolz, C. Debuschewitz, W. Belzig, and E. Scheer \\ Department of Physics, University of Konstanz, D-78457 Konstanz, Germany \\ (Received 26 April 2010; revised manuscript received 21 October 2010; published 12 September 2011)
}

\begin{abstract}
An experimental study of the proximity effect of superconductor-normal metal films with the help of lowtemperature scanning tunneling spectroscopy is reported. The behaviors of bilayers of the noble metals gold and silver in contact with the superconductor aluminum are compared for various thicknesses of the normal metal. Although the normal conducting properties of $\mathrm{Au}$ and $\mathrm{Ag}$ are very similar to each other, the measured differential conductance spectra from which the quasiparticle density of states is deduced differ markedly. While the behavior of the Al/Ag system follows the quasiclassical theory of the proximity effect for diffusive systems, differences exist for the Al/Au system. The absolute value of the induced minigap in Au is larger than predicted by theory, and its suppression with increasing temperature is weaker. These observations are quantitatively accounted for by including a finite interaction parameter for $\mathrm{Au}$ of $\left(N_{0} V\right)_{\mathrm{Au}}=0.10 \pm 0.03$. The third investigated metal is palladium, which is close to ferromagnetism. The method presented here enables one to detect small superconducting correlations by investigating a spectroscopic property rather than the supercurrent or the critical temperature.
\end{abstract}

DOI: 10.1103/PhysRevB.84.104516

PACS number(s): 74.78.-w, 74.25.F-, 74.45.+c, 74.55.+v

\section{INTRODUCTION: ELECTRON-PHONON COUPLING AND PROXIMITY EFFECT}

Electron-phonon interaction is omnipresent in all metals and strongly influences several prominent phenomena, such as the resistivity of metals or the heat conductivity, and is of crucial importance for superconductivity. It is, however, not straightforward to determine quantitatively because competing effects like magnetic ordering or Coulomb interaction may cover the effects of electron-phonon interaction. In superconducting systems, the electron-phonon coupling can in principle be determined from the critical temperature using the McMillan formula or approximations of this formula. ${ }^{1}$ Owing to their relative simplicity, the noble metals serve as benchmark systems for band-structure calculations, and particular interest exists in the experimental determination of their electronphonon coupling strength. However, the noble metals are not superconducting in the experimentally accessible temperature range. But superconducting correlations may be induced using the proximity effect $(\mathrm{PE})$ with a bulk superconductor.

The electron-phonon coupling constant $\lambda$ of $\mathrm{Au}$ was calculated from first principles using band-structure calculations with the value of $\lambda_{\mathrm{Au}}=0.17$. $^{2}$ This is the highest value among the noble metals and one of the highest of all elementary nonmagnetic and nonsuperconducting metals. Experimentally, no intrinsic superconductivity has been found so far for $\mathrm{Au},{ }^{3-5}$ presumably because of magnetic impurities, which suppress very efficiently coherent supercurrents. ${ }^{6}$ However, several Au-based alloys with measurable critical temperatures do exist, ${ }^{7}$ and several experiments have been conducted to enhance $\lambda_{\mathrm{Au}}$ by pressure. ${ }^{8}$

In the case of Pd, spin fluctuations can give a repulsive contribution to the electron-electron interaction. ${ }^{9}$

PE denotes the induction of superconducting properties into a normal metal in contact with a superconducting one. Although the PE was studied thoroughly in the 1960s, pioneered by the work of the Orsay group on superconductivity, ${ }^{10}$ it has experienced a rediscovery during the last two decades, initiated by the possibility of probing superconducting properties at length scales shorter than the quantum coherence length of the electron transport in the normal state. ${ }^{11-17} \mathrm{It}$ has been understood that the range in which the PE is active depends on the coherence length $\xi$, the normal conducting properties (elastic mean free path and conductivity) of both the superconductor and the normal conductor, the dimensionality and dimensions of the samples, the transport regime (diffusive or ballistic), and the properties of the interface between both metals. When the electron transport has a diffusive character, the PE can be described by a quasiclassical theory ${ }^{18}$ using the Usadel equations. ${ }^{19}$ One of the most prominent physical quantities that reveals modifications due to the $\mathrm{PE}$ is the quasiparticle density of states (DOS), which can be probed with high precision by analyzing the spectra of tunnel contacts. ${ }^{12}$ Tunable tunnel contacts can be arranged with the help of a scanning tunneling microscope (STM) in its spectroscopy mode, ${ }^{20}$ i.e., by recording the differential conductance of a given tunnel contact. When the DOS of the superconductor is known quantitatively, intrinsic properties of the normal metal, such as spin-flip or inelastic scattering in the normal metal, spin polarization in ferromagnetic metals, ${ }^{21,22}$ as well as geometry dependence or magnetic flux, may be studied. $^{23}$

\section{THEORETICAL BACKGROUND AND EXPERIMENTAL CONCEPT}

According to McMillan's theory, ${ }^{1}$ the critical temperature of a superconductor is determined by its Debye temperature $\Theta_{D}$, the electron-phonon coupling constant $\lambda$, and the Coulomb interaction strength $\mu^{*}$ in the following way:

$$
T_{c}=\frac{\Theta_{D}}{1.45} \exp \left[-\frac{1+\lambda}{\lambda-\mu^{*}(1+0.62 \lambda)}\right] .
$$

When assuming weak interaction, the critical temperature of a superconductor can be approximated to the 
Bardeen-Cooper-Schrieffer (BCS) formula

$$
T_{c}=1.13 \frac{\hbar \omega_{c}}{k_{B}} \exp \left(-\frac{1}{N_{0} V}\right),
$$

where $\omega_{c}$ is the cutoff frequency, which is of the order of the Debye frequency; $N_{0}$ is the DOS; and $V$ is the potential for electron-phonon scattering. ${ }^{24}$ In this approximation, a correlation can be established between the electron-phonon coupling constant $\lambda$, which appears in the McMillan formula, and the pairing parameter $N_{0} V=\lambda-\mu^{*}$ of the BCS theory. For simplicity and because the $\mu^{*}$ values of the systems under study here cannot be determined independently, we restrict our analysis to the BCS theory, which has been proven to account for the superconducting properties of $\mathrm{Al}$ even quantitatively. To avoid confusion, we will use the notation $N_{0} V$ throughout the manuscript and we will make no statements about $\lambda$.

We use here the comparison of the two noble metals $\mathrm{Ag}$ and $\mathrm{Au}$ for determining the pairing parameter $\left(N_{0} V\right)_{N}$ of Au. $\left(N_{0} V\right)_{N}$ is the crucial quantity for the formation of pair correlations, according to the conventional pairing mechanism first described in the BCS theory. The limits of validity of the approach are revealed by studying the PE between $\mathrm{Al}$ and the strong paramagnet $\mathrm{Pd}$, which is close to the transition to ferromagnetism. In this system, spin fluctuations introduce an additional pair-breaking mechanism. Electronphonon coupling is one of the most fundamental quantities of superconductivity, and much effort is taken to measure and to calculate it for elements as well as for compounds that may possibly be superconductors.

The determination of $N_{0} V$ via resistive measurements of the critical temperature is difficult, as long-range supercurrents are easily suppressed by extrinsic influences. In the $T_{c}$ formula (2), $N_{0} V$ enters in the denominator of the argument of an exponential, resulting in a strongly nonlinear dependence. Here, the PE offers a more practical way to determine $\left(N_{0} V\right)_{N}$ in normal metals, because the width of the proximity-induced minigap is influenced by a finite value of $\left(N_{0} V\right)_{N}$ in a much more direct way than the critical temperature. When considering a one-dimensional diffusive system consisting of a superconductor $S$ with diffusion coefficient $D_{S}$ and a normal metal with a diffusion coefficient $D_{N}$ and a finite $\left(N_{0} V\right)_{N}$, the Usadel equation for the so-called pairing angle reads ${ }^{19}$

$$
\begin{aligned}
\frac{D_{N / S}}{2} \frac{\partial^{2} \theta(x)}{\partial x^{2}}= & -i E \sin \theta(x)-\Delta_{N / S}(x) \cos \theta(x) \\
& +\Gamma_{s f} \sin [2 \theta(x)] .
\end{aligned}
$$

The pairing angle is related to retarded and anomalous Green's functions via $G_{R}(E, x)=\cos \theta(x)$ and $F_{R}(E, x)=\sin \theta(x)$, respectively. $\Gamma_{s f}$ is the spin-flip scattering rate, which accounts for dephasing due to scattering at magnetic impurities. Finally, the influence of spin excitations (paramagnons) might play a role in the $\mathrm{Al} / \mathrm{Pd}$ system. They can be described by including an additional parameter $\lambda_{s f} .{ }^{25}$ This will be discussed in detail in Appendix B. The pair potentials have to be determined self-consistently via

$$
\begin{aligned}
\Delta_{N / S}(x)= & \frac{\left(N_{0} V\right)_{N / S}}{2} \int_{0}^{k_{B} \Theta_{D, N / S}} d E \sin [\theta(E, x)] \\
& \times \tanh \left(\frac{E}{2 k_{B} T}\right) .
\end{aligned}
$$

The integral is taken over the energy range from zero up to the Debye energy $k_{B} \Theta_{D, N / S}$ of the $N$ and the $S$ metal, respectively. The values used here are given in Table I. The influence of the cutoff frequency on the spectra and the apparent minigap is discussed in Appendix A. From the self-consistent solution of the Usadel equation, the DOS of the normal metal can be deduced and reads

$$
N(E, x)=\operatorname{Re} \cos [\theta(E, x)] .
$$

The pair potential induced by a superconductor into a normal metal is fairly large, as it is directly proportional to the normal metal coupling constant and not exponentially suppressed like $T_{c}$ in the corresponding bulk metal. Hence, the $\left(N_{0} V\right)_{N}$ value enters linearly into an additional term of the spectral gap of the DOS. We use this fact for determining the pairing interaction in gold by comparing experimental spectra of proximity structures, in which aluminum serves as a superconductor, to the quasiclassical theory.

TABLE I. Electronic properties of the metal films under investigation. The Fermi velocity $v_{F}$, the electron density $n$, the effective mass

\begin{tabular}{|c|c|c|c|c|c|c|c|c|c|c|c|}
\hline Element & $\begin{array}{c}v_{F} \\
\left(10^{6} \mathrm{~m} / \mathrm{s}\right)\end{array}$ & $\begin{array}{c}n \\
\left(10^{28} \mathrm{~m}^{-3}\right)\end{array}$ & $m^{*} / m$ & $\begin{array}{c}l_{e p} \\
(\mathrm{~nm})\end{array}$ & $\begin{array}{c}l \\
(\mathrm{~nm})\end{array}$ & $\begin{array}{c}\sigma \\
\left(10^{7} \mathrm{~S} / \mathrm{m}\right)\end{array}$ & $\begin{array}{c}D \\
\left(\mathrm{~m}^{2} / \mathrm{s}\right)\end{array}$ & $\begin{array}{c}\xi \\
(\mathrm{nm})\end{array}$ & $\gamma_{\mathrm{Al}}$ & RRR & $\begin{array}{l}\Theta_{D} \\
(\mathrm{~K})\end{array}$ \\
\hline $\mathrm{Au}$ & 1.40 & 5.90 & 1.1 & 50 & 56 & 6.1 & 0.026 & 210 & 0.657 & 2.1 & 165 \\
\hline $\mathrm{Ag}$ & 1.39 & 5.86 & 1.1 & $58^{\mathrm{a}}$ & 56 & 6.1 & 0.026 & 209 & 0.660 & 2.1 & 215 \\
\hline $\mathrm{Pd}^{\mathrm{b}}$ & 0.2 & - & - & - & 3.3 & 0.72 & $2.2 \times 10^{-4}$ & 19 & 0.871 & - & 274 \\
\hline $\mathrm{Pd}^{\mathrm{c}}$ & 0.56 & - & - & - & 9.2 & 0.72 & $1.7 \times 10^{-3}$ & 54 & 0.312 & - & 274 \\
\hline $\mathrm{Al}$ & 2.03 & 18.1 & 1.4 & - & 68 & 12 & 0.046 & 279 & - & - & 428 \\
\hline
\end{tabular}
$m^{*} / m$, and the electron-phonon scattering length at room temperature $l_{e p}$ are taken from literature. The elastic mean free path $l$ was determined from the residual resistivity ratio (RRR), equal to $R(300 \mathrm{~K}) / R(4 \mathrm{~K})$, of films or wires with the same thickness of the $N$ metal but a thin $S$ layer. The electronic diffusion constant $D$, the conductivity $\sigma$, and the interface parameter $\gamma_{\mathrm{Al}}$ were deduced from these values using the Drude formula (for details, see text); the conductivities $\sigma_{\mathrm{Al}}$ of an $\mathrm{Al}$ wire and $\sigma_{\mathrm{Pd}}$ of a Pd wire were directly measured. The last column contains the values of the Debye temperature used for the fitting.

${ }^{a}$ Calculated using values for RRR and $\rho_{0}$ from Ref. [28].

${ }^{\mathrm{b}}$ Assuming band properties of d electrons.

${ }^{\mathrm{c}}$ Assuming band properties of s electrons. 


\section{EXPERIMENTAL}

The DOS was measured using a very low-temperature STM, described in Ref. [27]. The STM is mounted on a ${ }^{3} \mathrm{He}$ cryostat. It comprises only one piezo tube for scanning and coarse approach, using slip and stick motion. We used electrochemically etched tungsten tips as counter electrodes. The experimental scheme is shown in Fig. 1. We chose thin diffusive films of aluminum in spite of their low critical temperature and small gap energy because Al fulfills best the predictions of BCS theory. Bulk $\mathrm{Al}$ has a critical temperature of $T_{c}=1.2 \mathrm{~K}$ and a gap energy of $\Delta=180 \mu \mathrm{eV}$, resulting in a ratio of $2 \Delta / k_{B} T_{C}=3.5$ and a DOS in almost perfect agreement with the predictions of the BCS theory. Its electronphonon coupling constant is rather isotropic, and the pairing parameter $N_{0} V$ has been calculated using first principles to amount to $\left(N_{0} V\right)_{\mathrm{Al}}=0.23$. Experimentally, it has been determined using the BCS formula to be $\left(N_{0} V\right)_{\mathrm{Al}}=0.17 .^{26}$

We investigated $S / N$ bilayer samples that were prepared using ultrahigh-vacuum metal evaporation at room temperature onto silicon substrates. First, Al (purity $99.999 \%$ ) was thermally evaporated with a rate of $\sim 1 \mathrm{~nm} / \mathrm{s}$, measured with a quartz sensor. Not more than 15 min later, the normal Ag, Au (purity $99.99 \%$ for both), or Pd (purity 99.95\%) were electron-beam deposited with rates between 0.1 and $0.2 \mathrm{~nm} / \mathrm{s}$ without breaking the vacuum of $p=10^{-7}$ to $10^{-6} \mathrm{~Pa}$. The thickness has been determined from the calibration of the quartz monitor, which has been verified regularly. We estimate the real thicknesses to be within $10 \%$ of the nominal value.

As interdiffusion between both metals may affect the interface properties, which in turn influence the PE, the samples are cooled down immediately (within three hours after deposition of the normal metal) to a temperature below $4 \mathrm{~K}$. We will discuss the influence of aging effects below. During cooldown, the temperature of the sample is kept slightly higher than that of the surroundings in order to minimize condensation of gas molecules and humidity.

$d I / d V$ spectra were derived using a lock-in amplifier. The direct-current-bias voltage was modulated with a lowfrequency alternating-current voltage with a root-mean-square amplitude of $U_{\text {mod }}=14 \mu \mathrm{V}$. The STM has a high-voltage resolution of approximately $20 \mu \mathrm{V}$ at base temperature, which
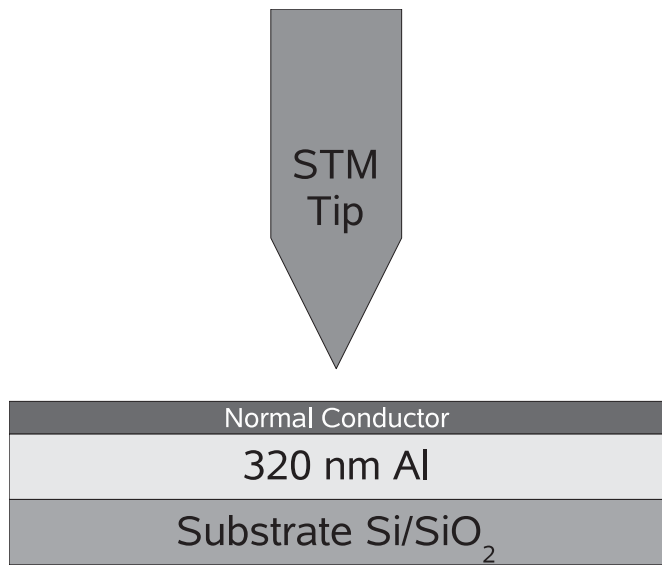

FIG. 1. A two-layer system consisting of a 320-nm-thick Al layer covered with normal metal is studied using an STM. was determined to be $270 \mathrm{mK}$ by fitting spectral data recorded on Al layers to the BCS theory. The signal was measured using a current-to-voltage amplifier with an amplification of $1 \mathrm{~V} / \mathrm{nA}$. The measuring time for one spectrum is approximately $1 \mathrm{~min}$. In the case of $\mathrm{Pd}$, the average over two to five spectra is shown. We did not observe significant contact-to-contact variations when scanning over the sample within the same cooldown. All spectra presented here were taken at a tunneling resistance in the order of $R_{T}=10 \mathrm{M} \Omega$. This resistance was chosen to have sufficient signal-to-noise ratio while avoiding distortions of the spectrum due to the presence of the counter electrode. Corrections due to Andreev reflection scale with $R_{T}^{-1} / G_{0}$, where $G_{0}=2 e^{2} / h$ is the conductance quantum. They are in the order of one permille for this resistance. The contact was formed by turning off the feedback loop at a current of $400 \mathrm{pA}$ and a voltage of $4 \mathrm{mV}$. For measurements at higher temperatures, the STM was allowed to stabilize for at least $5 \mathrm{~min}$ at each temperature. Furthermore, all spectra are normalized with respect to the ohmic conductance measured at voltages outside the superconducting gap. Voltage offsets were subtracted using the symmetry of the spectra.

\section{RESULTS AND DISCUSSION}

\section{A. Sample characterization measurements}

The measured spectra for different thicknesses $d_{N}$ of the normal metals are shown in Fig. 2. These measurements were performed at the base temperature of $270 \mathrm{mK}$. We used the quasiclassical theory of the $\mathrm{PE}^{29}$ to calculate the DOS for these samples. Because the thermal energy $k_{B} T \approx 100 \mu \mathrm{eV}$ is similar to the applied bias voltage, the influence of the Fermi distribution of electrons was taken into account for calculating $d I / d V$ spectra for comparison with the measured spectra. For our experiment, the dirty limit of the theory is of interest, meaning $\xi_{N} \gg l$ with $\xi_{N}=\sqrt{\hbar D_{N} / 2 \Delta}$ and $l$ being the superconducting coherence length and the elastic mean free path, respectively. The thickness of the Al layer is between 320 and $360 \mathrm{~nm}$ in all samples, which is thicker than the coherence length $\xi_{\mathrm{Al}}=279 \mathrm{~nm}$ using the values $D=0.046 \mathrm{~m}^{2} / \mathrm{s}$ for the diffusion constant and $l=68 \mathrm{~nm}$ for the elastic mean free path (deduced from the resistivity of a wire).

An important parameter describing the difference of electronic properties at the interface is

$$
\gamma_{\mathrm{Al}}=\frac{\sigma_{N}}{\sigma_{\mathrm{Al}}} \sqrt{\frac{D_{\mathrm{Al}}}{D_{N}}},
$$

where $\sigma_{N}$ and $\sigma_{\mathrm{Al}}$ are the conductivities and $D_{N}$ and $D_{\mathrm{Al}}$ are the diffusion constants in the normal metal and in the superconductor, respectively. Mismatches between the Fermi momenta of $S$ and $N$ result in an imperfect transmission coefficient $\mathcal{T}$ of the interface, giving rise to a finite interface resistance $R_{B}$. It is usually accounted for by a barrier parameter $\gamma_{B}$, which is used as a fitting parameter, ${ }^{30}$ because an analytical formula for $\gamma_{B}$ has been given in the tunnel limit only. ${ }^{31,32} \gamma_{B}$ enters through the boundary conditions into the calculation. To get an estimate of this parameter, we use

$$
\gamma_{B}=\frac{R_{B} \sigma_{N}}{\xi_{N}} \approx \frac{2}{3} \frac{1-\mathcal{T}}{\mathcal{T}} \frac{l_{N}}{\xi_{N}}
$$



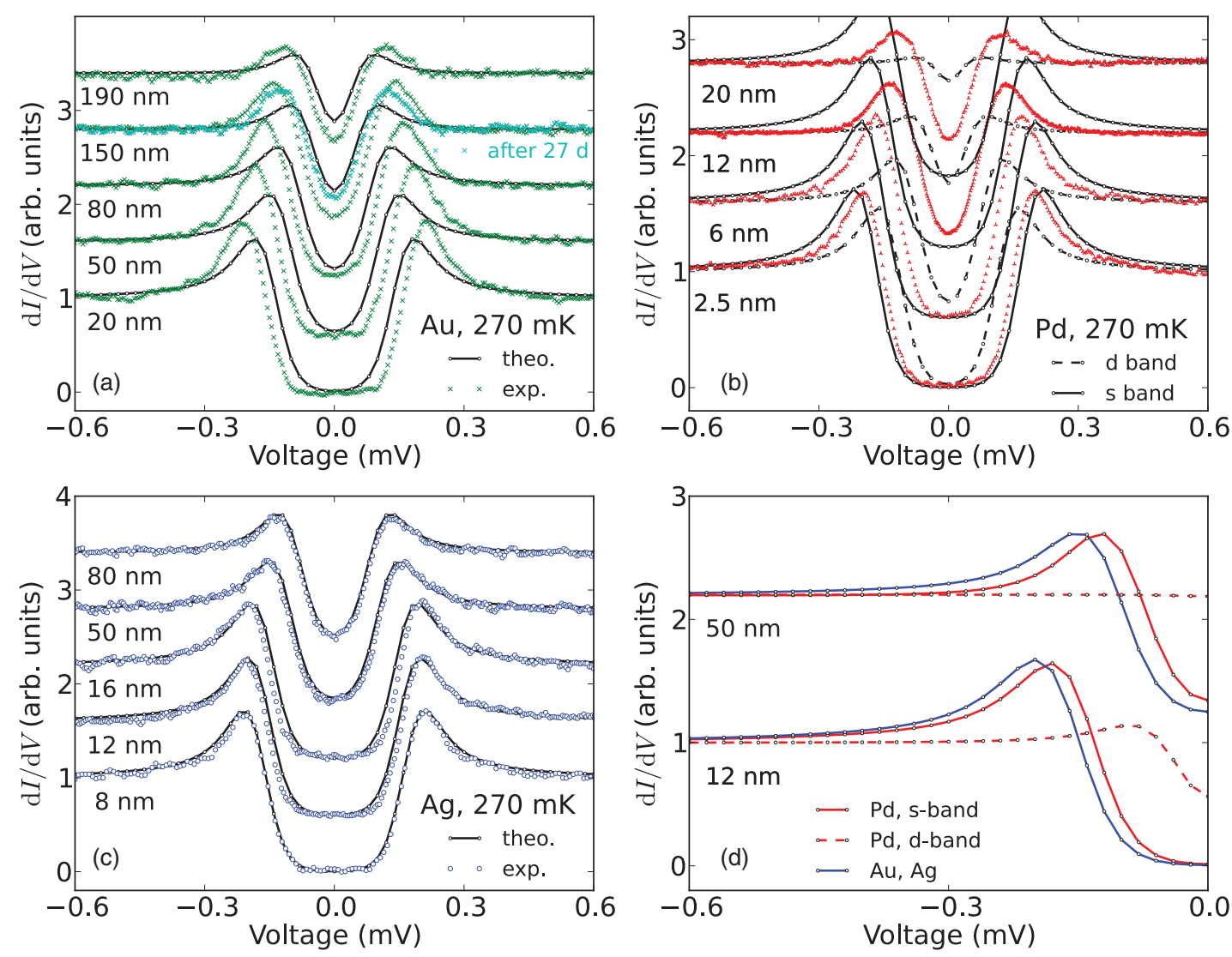

FIG. 2. (Color online) Spectra taken at $270 \mathrm{mK}$ on different materials and different thicknesses: (a) $\mathrm{Al} / \mathrm{Au},(\mathrm{b}) \mathrm{Al} / \mathrm{Pd}$, and (c) $\mathrm{Al} / \mathrm{Ag}$. Lines represent calculated DOS according to the quasiclassical theory for diffusive systems using the experimentally determined values of $l$ and $\sigma$ for $\mathrm{Au}$ and $\mathrm{Ag}$. (a) Spectra measured on 150-nm Au on Al directly after sample preparation and after $27 \mathrm{~d}$ at ambient conditions. For Pd, we show the results of the calculation assuming that either the $\mathrm{d}$ or s electrons are responsible for the PE. (d) Calculated DOS for Au, Pd, and Ag with a thickness of 12 and $50 \mathrm{~nm}$, respectively. Curves are shifted vertically for clarity.

and apply the ballistic result $\mathcal{T}=4 k_{F, N} k_{F, S} /\left(k_{F, N}+k_{F, S}\right)^{2}$ as the upper limit for $\mathcal{T}$. Taking the values listed in Table I, we expect for the interface parameter that $\gamma_{B(\mathrm{Al} / \mathrm{Au})}=\gamma_{B(\mathrm{Al} / \mathrm{Ag})} \approx$ 0.02 . For Pd, the values of $\gamma_{B, \mathrm{Al} / \mathrm{Pd}}$ assuming $\mathrm{s}$ and d electrons are $\gamma_{B, \mathrm{Al} / \mathrm{Pd}} \approx 0.02$ and $\gamma_{B, \mathrm{Al} / \mathrm{Pd}} \approx 0.13$.

For $\mathrm{Ag}$ and $\mathrm{Au}$, we determine the conductivities and diffusion constants from $\mathrm{RRR}=R(300 \mathrm{~K}) / R(4 \mathrm{~K})$ of film samples. Using the Drude formula $\sigma=n e^{2} \tau / m$, one obtains $\sigma$ assuming the literature values for $v_{F}$. We used samples that were fabricated in the same way as the bilayer samples but with only $10 \mathrm{~nm}$ of $\mathrm{Al}$ to obtain similar growth conditions for the normal metals $\mathrm{Ag}$ and $\mathrm{Au}$ on top. ${ }^{49}$ The RRR values are listed in Table I. From RRR $\simeq\left(\rho_{e p}+\rho_{0}\right) / \rho_{0}=l / l_{e p}+1$, one can estimate the mean free path $l$ if the electron-phonon scattering length $l_{e p}$ is known. The determination of $l_{\mathrm{Pd}}$ using the Drude formula is not straightforward because the electronic structure of Pd differs markedly from the single-band, quasifree electron model. We therefore apply the Einstein formula $\sigma=n\left(E_{F}\right) e^{2} D$ for the determination of the diffusion constant from the measured conductivity. ${ }^{33}$ The gap in the superconductor $\Delta_{0}=195 \mu \mathrm{eV}$ was determined by fitting the theory for a BCS superconductor to the data recorded on an Al layer without normal metal on top. This value is somewhat higher than the bulk value of $\Delta_{0}=180 \mu \mathrm{eV}$ but in agreement with the usual observation in thin films or wires. ${ }^{11}$ We use our experimentally observed gap value for analyzing the spectra of the bilayers. The spectrum is shown in Fig. 4(d).

\section{B. Analysis of the proximity effect}

We now turn to the tunneling spectroscopy measurements of the $S / N$ bilayers. Figure 2 summarizes the spectra measured at our lowest measuring temperature of $270 \mathrm{mK}$ for the $\mathrm{Al} / \mathrm{Au}$ [panel (a)] and Al/Ag [panel (c)] bilayers with varying thicknesses. In addition, we show spectra measured on $\mathrm{Al} / \mathrm{Pd}$ bilayers [panel (b)]. In panel (a), two experimental curves for the thickness $d=150 \mathrm{~nm}$ are given. The green one has been measured on a fresh sample directly after sample preparation; the light blue one has been measured after 27-d storage under ambient conditions. We argue that interdiffusion took place that altered the interface properties between $\mathrm{Al}$ and $\mathrm{Au}$. In what follows, we will consider spectra obtained on as-prepared samples only. Interdiffusion and aging effects will be discussed further in Sec. IV.C. The amplitudes of the differential conductance traces $d I / d V$ normalized to their values far outside the gap $(|V| \gg \Delta)$ are offset vertically for clarity. The spectra show a pronounced minigap with decreasing width for increasing thickness of the normal metal. The black lines correspond to the calculated DOS according to Eq. (3) using the parameters given in Table I, assuming zero 
pair interaction in the $\mathrm{N}$ metal: $\left(N_{0} V\right)_{N}=0$, with zero $\lambda_{s f}$. We note that the lines are mere calculations; no fitting to the data has been performed. Furthermore, we assumed a nonresistive interface, $\gamma_{B}=0$. Since the normal conducting properties of our $\mathrm{Au}$ and $\mathrm{Ag}$ films are almost equal, very similar spectra are expected for both metals.

In the case of $\mathrm{Pd}$, two series of theory curves are given corresponding to the two dominating bands, the $\mathrm{s}$ band and the $d$ band crossing the Fermi energy. The model assuming the $\mathrm{s}$ band fits satisfactorily at low thicknesses, while it fails to describe the spectra at higher thicknesses. The d-band description underestimates the gap width for all thicknesses. At a first glimpse, one might take this discrepancy as an indication that the s-band electrons couple better to the Cooper pairs, as assumed in Refs. [33,35]. However, we will show in Appendix B that equally good agreement can be obtained between experiment and theory for both bands by adjusting the free parameters $\Gamma_{s f}$ and $\left(N_{0} V\right)_{\mathrm{Pd}}$ and the paramagnon contribution $\lambda_{s f}$. Because of this complexity, it is not possible to find unambiguously a value of $\left(N_{0} V\right)_{\mathrm{Pd}}$. In Appendix B, we discuss the extent to which our results are in accordance with the literature. For the rest of the main manuscript, we will therefore restrict ourselves to considering the systems $\mathrm{Al} / \mathrm{Au}$ and $\mathrm{Al} / \mathrm{Ag}$.

Summarizing, our main findings are the following: 1 . The low-temperature spectra recorded for the $\mathrm{Al} / \mathrm{Ag}$ bilayers are in good agreement with the "simple" Usadel theory without using fitting parameters. ${ }^{18}$ 2. For the $\mathrm{Al} / \mathrm{Au}$ system, the experimental values of the minigap are larger than those calculated from theory for all samples. We will show below that these findings hold for higher temperatures as well. 3 . The degree of agreement or disagreement between theory and experiment has no systematic dependence on the layer thickness, even down to the smallest ones in the order and even below the elastic mean free path. This observation is an example of the weak dependence of the spectrum and the minigap on the elastic mean free path as predicted by Pilgram et al. $^{34}$

We first discuss whether finite spin-flip scattering or interface resistance would lift the discrepancy for the Al/Au system. As an example, we replot in Fig. 3 the $d I / d V$ $(T=270 \mathrm{mK})$ curve of the Al/Au bilayer with $d=50 \mathrm{~nm}$ together with the Usadel result with $\gamma_{B}=\Gamma_{s f}=0$ and as a shaded area the possible shapes obtained for combinations of $0<\gamma_{B}<3$ and $0<\Gamma_{s f}<0.2 \Delta$. Small values of $\gamma_{B}$ give rise to a slightly enhanced gap in the differential conductance and an anomaly at the gap edge that is sharper but not by a sufficient amount. When exceeding $\gamma_{B}=0.9$, the height of the anomaly starts to decrease again. $\Gamma_{s f}$ reduces the minigap and results in a rounded shape of the DOS for all values. Obviously, no good agreement with the theory is obtained. Higher values can be excluded because this would result in even stronger reduction of the gap and the amplitude close to the gap edge. The same conclusion was drawn from the spectra obtained for the other thicknesses and higher temperatures.

As a next step, we include an attractive pair interaction in $\mathrm{Au}, I P=\left(N_{0} V\right)_{N} /\left(N_{0} V\right)_{S}$, as the only free-fitting parameter [see Fig. 4(a)]. In Fig. 4(b), we plot the mean-square deviation $\chi^{2}$ as a function of $I P$ for all samples obtained at $T=270 \mathrm{mK}$ and the average over all thicknesses. Values of $I P=0.6 \pm 0.15$

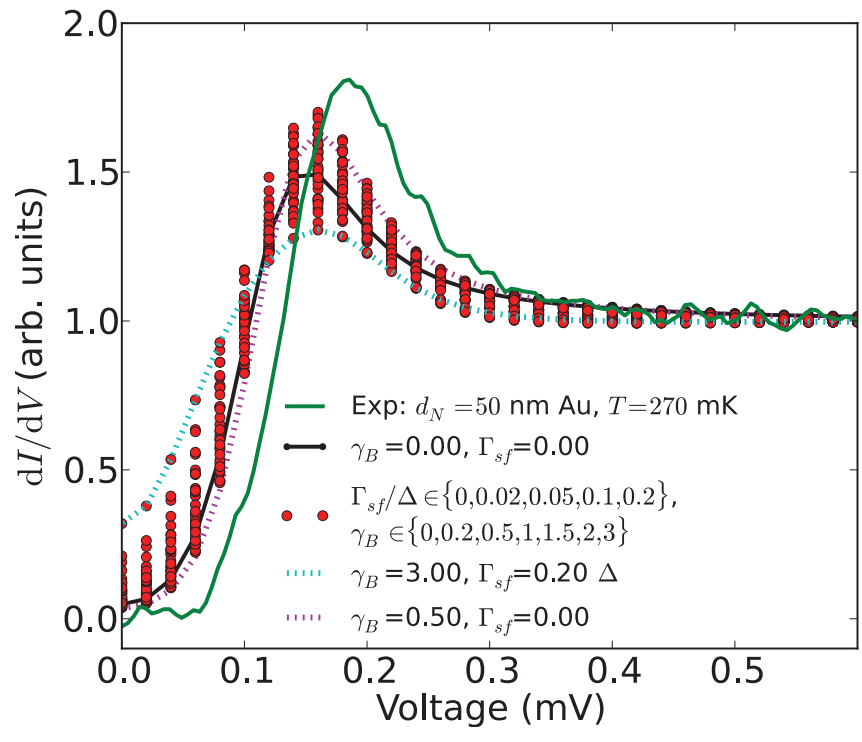

FIG. 3. (Color online) Spectrum of the Al/Au bilayer with $d_{N}=$ $50 \mathrm{~nm}$ compared with expected shapes for various combinations of $\gamma_{B}$ and $\Gamma_{s f}$ but with $I P_{\mathrm{Au} / \mathrm{Al}}=\left(N_{0} V\right)_{\mathrm{Au}} /\left(N_{0} V\right)_{\mathrm{Al}}=0$.

describe the experimentally obtained spectra well. The value of $\chi^{2}$ is reduced by a factor of more than 3 upon inclusion of $I P$. If we assume $I P=0.6$, only very slight improvement of the fit can be obtained when including $\gamma_{B}$ as a fit parameter. Including also $\Gamma_{s f}$ results in $\Gamma_{s f}=0$ as the best-fit value. Because of the nonmonotonous dependence of the minigap on $\gamma_{B}$, meaningful fitting is restricted to $\gamma_{B}<1$ because for higher values the influence of increasing $\gamma_{B}$ will be compensated by increasing $I P$. The result of this procedure is shown in Fig. 4(b). As found before, there is a small but not systematic dependence on $d_{N}$. The ensemble of data is best described by $I P_{\mathrm{Al} / \mathrm{Au}}=0.6 \pm 0.15$. In the case of $I P=0.6$, the dependence of $\chi^{2}$ on $\gamma_{B}$ is very weak, so we cannot determine it from this set of data.

Repeating the same procedure for the $\mathrm{Al} / \mathrm{Ag}$ systems results in the following best-fit parameters: $I P_{\mathrm{Al} / \mathrm{Ag}}=0.16 \pm 0.08$, $\gamma_{B}=0 \pm 0.1$, and $\Gamma_{s f}=(0.05 \pm 0.05) \Delta$. The exact determination of $\Gamma_{s f}$ from our data is not possible, because up to $\Gamma_{s f}=0.1 \Delta$ higher values can be compensated by higher values of $\left(N_{0} V\right)_{\mathrm{Ag}}$. We note that with these best-fitting values the mean-square deviation is reduced by only $25 \%$ compared with the simple Usadel theory without any free parameters. The value $I P_{\mathrm{Al} / \mathrm{Ag}}=0.16 \pm 0.08$ hence sets an upper bound for the electron-phonon coupling strength in $\mathrm{Ag}$.

These findings are accomplished by the data recorded at a higher temperature up to the respective critical temperature of the bilayer systems shown in Fig. 5. The critical temperature is determined as the temperature at which the spectrum appears flat with no systematic reduction at low bias. The error bars are given by the temperature difference at which the spectra were taken.

Also, at elevated temperature the experimentally observed minigap for the $\mathrm{Al} / \mathrm{Au}$ system is larger than expected by the simple diffusive theory [see Fig. 5(a)]. The minigap vanishes at a higher temperature than expected from the theory. We also fitted the thickness dependence of the critical temperature with 

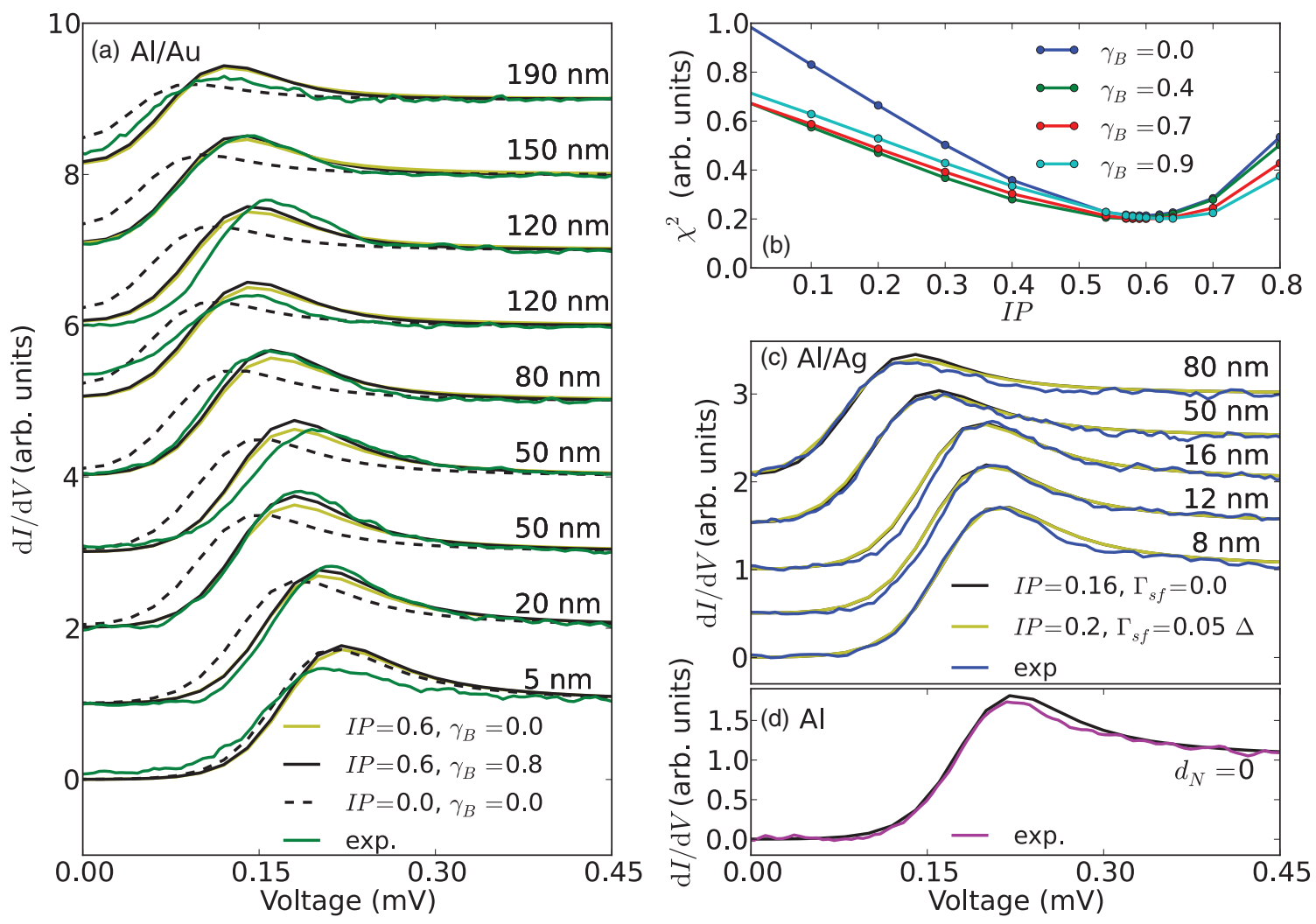

FIG. 4. (Color online) (a) Spectra of Al/Au bilayers for various Au thicknesses and best fits to the Usadel equation with $I P=0.6$ and $\gamma_{B}=0.8$; also shown are calculated spectra with $\gamma_{B}=0$ and $\gamma_{B}=I P=0$ for comparison. The experimental curve for $d=150 \mathrm{~nm}$ has been measured on the as-prepared sample. (b) Mean-square deviation $\chi^{2}$ as a function of $I P$. (c) Same as panel (a) but for the Al/Ag system. (d) The spectrum of Al without normal metal on top $\left(d_{N}=0\right)$.

$I P$ and $\gamma_{B}$ as free parameters. We find that these data are best described by $I P=0.6$ and $\gamma_{B}=0.8$. For values of the IP up to $I P=0.8$, very similar agreement of theory and experiment can be achieved by reducing $\gamma_{B}$. These parameter combinations, however, result in a very poor description of the spectra. Spectra calculated for higher temperatures with the best-fitting values found from the low-temperature spectra and the critical temperature $\left(I P=0.6\right.$ and $\left.\gamma_{B}=0.8\right)$ are shown in Fig. 5. For $\mathrm{Al} / \mathrm{Ag}$, the spectra agree well with the results of the Usadel equation [see Fig. 5(b)]. Only a very slight improvement was obtained when using $I P_{\mathrm{Al} / \mathrm{Ag}}=0.16$ and $\gamma_{B}=\Gamma_{s f}=0$.

Summarizing, we find that for the $\mathrm{Al} / \mathrm{Au}$ system the "simple" theory, i.e., without additional IP, systematically underestimates the minigap width and the critical temperature while it well describes the data for Ag. Yet, the agreement is improved by taking into account a relatively small $I P_{\mathrm{Al} / \mathrm{Ag}}=$ 0.16 . A striking observation is of the systematic difference between the behavior of the $\mathrm{Al} / \mathrm{Ag}$ and $\mathrm{Al} / \mathrm{Au}$ systems. It is striking because the normal-conducting properties of both normal metals are almost equal. However, interface effects might be different in both systems and give rise to differing PE. This is suggested by the necessity to include a finite $\gamma_{B}=0.8$ in the case of $\mathrm{Au}$, while $\gamma_{B}=0$ gave the best agreement for Ag. In Sec. IV.C, we will present control experiments that suggest that although interface effects are present they do not explain the enhanced PE observed in Al/Au. Finally, the same trend of an enhanced minigap in Au on top of the superconductor $\mathrm{Nb}$ had been observed before. ${ }^{36}$ Because of the discrepancy of the spectrum of pure $\mathrm{Nb}$ from the BCS shape, a detailed comparison with the theory was not possible in that experiment.

It has been suggested before that gold becomes superconducting at a critical temperature $T_{c, \text { Au }}$ between 100 (Ref. [4]) and $200 \mu \mathrm{K}$ (Refs. [37,38]). These estimates were derived from different types of experiments and used the BCS formula for $T_{c, \mathrm{Au}}$. Hoyt and Mota ${ }^{37}$ extrapolated the critical temperature measured for Au alloys to pure Au. Using the $\mathrm{BCS}$ relation (2), this corresponds to an interaction parameter of $\left(N_{0} V\right)_{\mathrm{Au}} \sim 0.073$. They also studied silver alloys but found by extrapolating their data a much smaller critical temperature $T_{c, \mathrm{Au}}=8 \times 10^{-10} \mathrm{~K}$. Buchal et al. investigated $\mathrm{Au}$ alloys as well as very pure $\mathrm{Au}$ samples. ${ }^{4}$ This experiment was performed at ultralow temperatures down to $38 \mu \mathrm{K}$ in a nuclear refrigerator. No signs of superconductivity could be observed in the pure $\mathrm{Au}$ samples, although the critical temperature was expected to be $100 \mu \mathrm{K}$. A possible explanation given by the authors is the extreme sensitivity of $T_{c, \mathrm{Au}}$ to 3-d-metal impurities.

The energy gap as a function of critical temperature in a normal metal in proximity to a superconductor as described in McMillan's tunneling theory ${ }^{39}$ was used by K. E. Gray $^{38}$ to determine the interaction parameter of Au. In contrast to our experiment, the tunneling of electrons from a superconductor into the normal side of an $\mathrm{Al} / \mathrm{Al}_{2} \mathrm{O}_{3} / \mathrm{Au}$ 

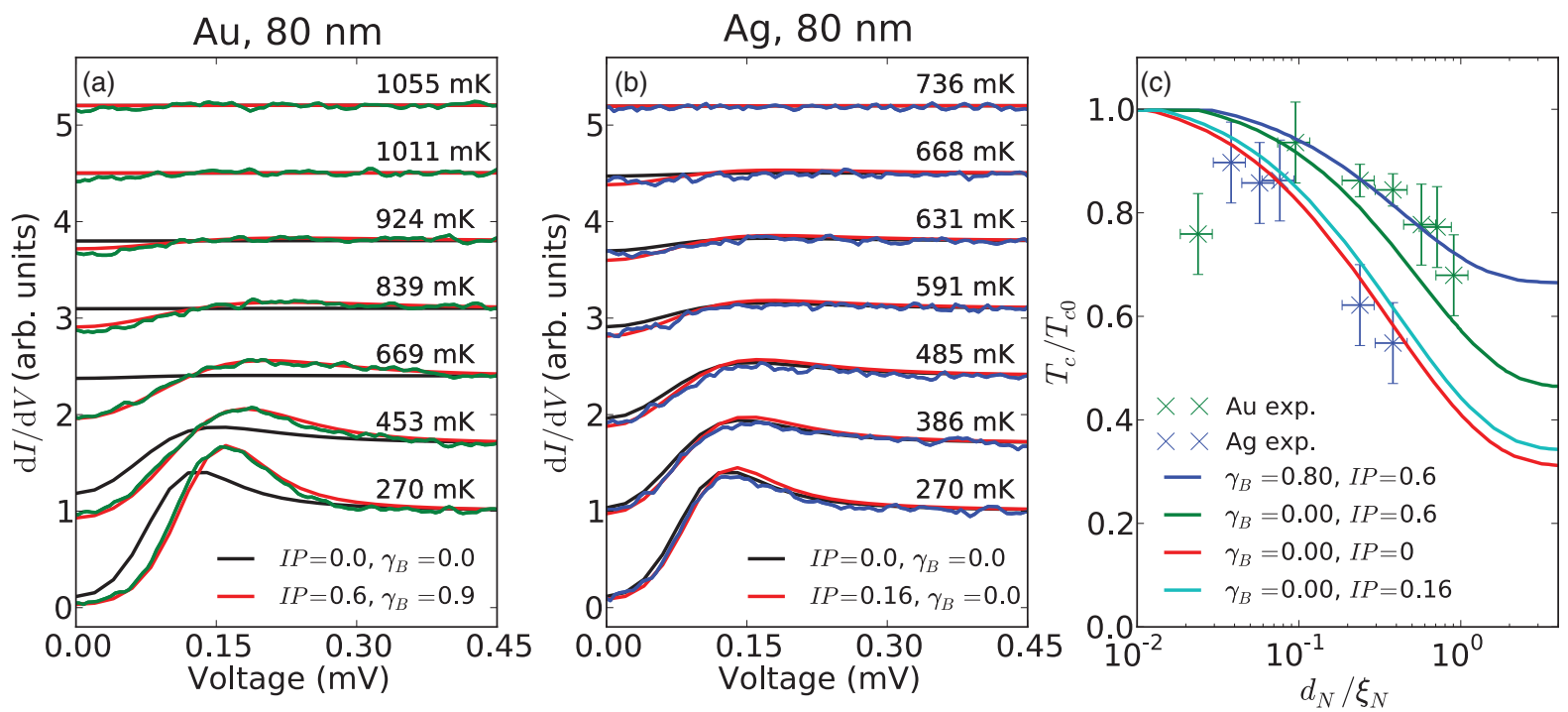

FIG. 5. (Color online) Examples of the temperature dependence of $d I / d V$ for one layer thickness of (a) $\mathrm{Al} / \mathrm{Au}$ and (b) $\mathrm{Al} / \mathrm{Ag}$. The green $\mathrm{Au})$ and blue $(\mathrm{Ag})$ lines denote the experimental data; black and red lines represent the theory. The curves are shifted vertically for clarity. (c) Plot of the critical temperature $T_{c}$ vs the logarithm of normal metal thickness in units of the coherence length $d_{N} / \xi_{N}$. The solid lines are calculated using the diffusive theory with interaction parameter $I P=0,0.16$, and $0.6 ; \Gamma_{s f}=0$; and best-fit values of $\gamma_{B}$ (see main text). Also shown is $I P=0.6$ with zero interface resistance $\left(\gamma_{B}=0\right)$.

contact was measured. The alumina film provided a weak barrier between $S$ and $N$. In that experiment, $\left(N_{0} V\right)_{\mathrm{Au}}=$ $0.072 \pm 0.004$ was derived. Using $\left(N_{0} V\right)_{\mathrm{Al}}=0.17,{ }^{26}$ one obtains the ratio $I P_{\mathrm{Au} / \mathrm{Al}}=\left(N_{0} V\right)_{\mathrm{Au}} /\left(N_{0} V\right)_{\mathrm{Al}}=0.43$, in good agreement with our experimental findings.

\section{Control experiments: interface effects}

The rather high value of $\gamma_{B}$, which is necessary to describe the $\mathrm{Al} / \mathrm{Au}$ spectra, points to a resistive interface between these metals. It is known that interdiffusion of Au into Al may occur when $\mathrm{Al}$ is evaporated on top of $\mathrm{Au}$ in very good vacuum conditions. As a result, the proximity spectra are smeared out. Under the vacuum conditions that we use here, and when first depositing $\mathrm{Al}$, a very thin alumina layer forms at the surface. This layer effectively hinders the interdiffusion of the subsequently deposited $\mathrm{Au}$ and results in well-defined proximity-induced minigaps. ${ }^{11,40}$ In one sample $(150 \mathrm{~nm} \mathrm{Au}$ on $\mathrm{Al}$ ), we observed a change of the spectra when measuring it again after having been stored for $27 \mathrm{~d}$ at ambient conditions [see Fig. 2]. The size of the gap was reduced. Indeed, for this old sample, the simple Usadel theory without $I P$ gave a rather good estimation of the gap at lowest temperature. However, the shape of the spectra at the gap edge and the data recorded at higher temperature still differ markedly from the simple theory. This observation of aging indicates that interdiffusion or other interface reorganizations could take place at these time scales. Nevertheless, since the aging effect reduces the gap, it cannot explain the observation of enhanced PE in the fresh samples. We performed control experiments in order to verify whether our observations are merely caused by an interface effect of the $\mathrm{Al} / \mathrm{Au}$ system; e.g., the formation of a superconducting $\mathrm{Au} / \mathrm{Al}$ alloy at the interface could result in a reduced effective $d_{N}$. We prepared a trilayer sample consisting of $320 \mathrm{~nm} \mathrm{Al}, 10 \mathrm{~nm}$ $\mathrm{Ag}$, and $70 \mathrm{~nm} \mathrm{Au}$. The silver interlayer is assumed to form the same interface with $\mathrm{Al}$ as in the $\mathrm{Al} / \mathrm{Ag}$ bilayer samples. Since $\mathrm{Ag}$ and $\mathrm{Au}$ are electronically very similar, we expect the $\mathrm{Ag} / \mathrm{Au}$ interface to give no particular contribution to the PE. The measured spectrum is shown in Fig. 6 in comparison with the Al/Ag80 and the Al/Au80 bilayer systems. The largest gap is observed for the trilayer system Al/Ag10/Au70. From this fact, we conclude that the enhancement of the minigap cannot be explained by a simple interface effect of $\mathrm{Al}$ and Au. Furthermore, one would expect the interface effect to be most prominent for thin layers of Au. Our experiment does not, however, reveal a systematic dependence of $I P_{\mathrm{Au} / \mathrm{Al}}$ on $d_{N}$.

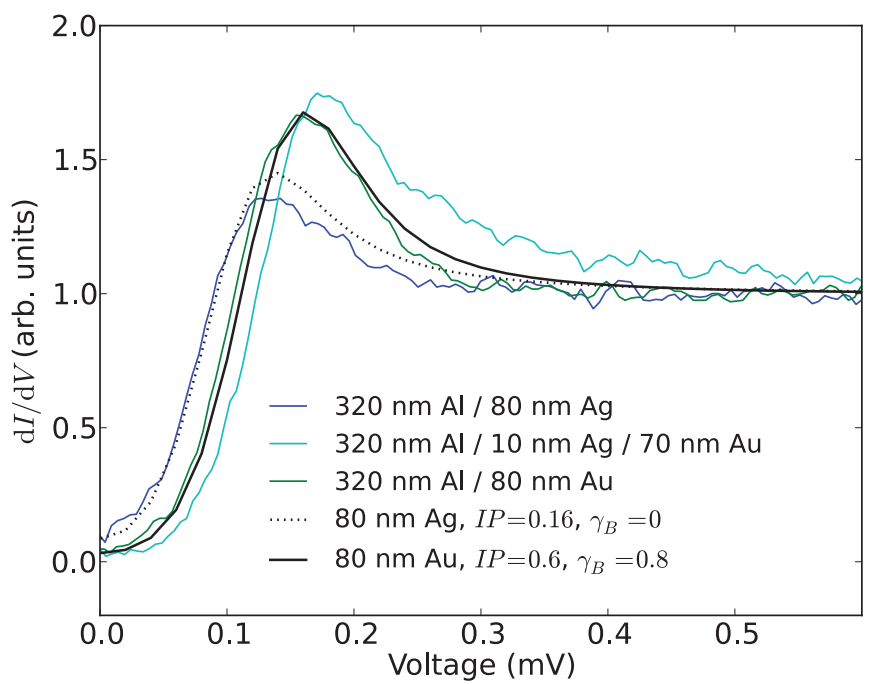

FIG. 6. (Color online) 80-nm Ag and 80-nm Au compared with trilayer 320-nm Al, 10-nm Ag, and 70-nm Au. Also shown are the calculated spectra for 80-nm Au or Ag. 


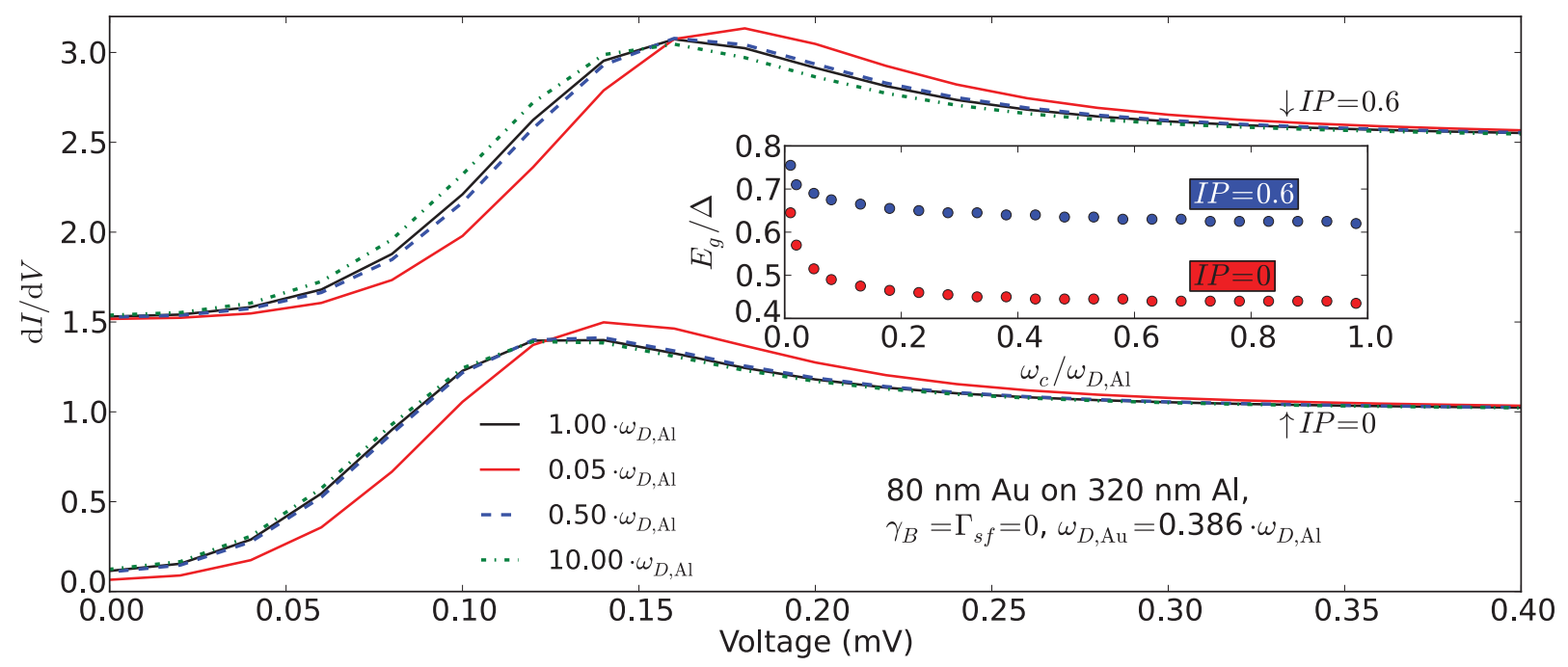

FIG. 7. (Color online) Influence of cutoff frequency $\omega_{c}$ in Eq. (4) on the calculated differential conductance. Inset: $E_{g}$ as a function of $\omega_{c}$.

\section{CONCLUSIONS}

We use the superconducting PE for determining the electron-phonon coupling strength of $\mathrm{Au}$ thin films. The quasiparticle DOS and the critical temperature of superconductor/normal metal bilayers have been measured by scanning tunneling spectroscopy. The energy gap and the critical temperature of $\mathrm{Al} / \mathrm{Au}$ bilayers are higher than expected from Usadel theory. The discrepancy can quantitatively be explained by taking into account an attractive pair interaction in $\mathrm{Au}$ of $\left(N_{0} V\right)_{\mathrm{Au}}=0.10 \pm 0.03$. Due to the exponential dependence of $T_{c}$ on the interaction strength this results in an expected critical temperature of $\mathrm{Au}$ in the range between $120 \mu \mathrm{K}$ and $85 \mathrm{mK}$, the mean value corresponding to $10 \mathrm{mK}$. These values are in agreement with previous predictions and extrapolations from experiments on Au-based alloys. The new aspect here is that the interaction parameter was determined directly for pure $\mathrm{Au}$ films and not by extrapolation from alloys. The behavior of the bilayers from $\mathrm{Al}$ with $\mathrm{Ag}$ are well described by the quasiclassical theory without electron-phonon interaction in the normal metal. Still, the agreement between the experimental and theoretical spectra can be improved by including a small interaction parameter that would correspond to a very small intrinsic critical temperature in the picokelvin regime. Finally, the system $\mathrm{Al} / \mathrm{Pd}$ reveals the necessity for a more sophisticated treatment including magnetic pair-breaking effects and a more complex description of the band structure of Pd.

\section{ACKNOWLEDGMENTS}

We thank C. Sürgers, H. v. Löhneysen, P. Leiderer, T. Kontos, N. O. Birge, and O. Millo for many useful discussions and H.-F. Pernau and V. Kunej for experimental help. This work was financially supported by the Research Network Functional Nanostructures of the Baden-Württemberg Stiftung and through the Deutsche Forschungsgemeinschaft (DFG). Part of this work has been performed in the framework of a Deutsch-Israelisches Projekt (DIP) funded by the Bundesministerium für Bildung und Forschung (BMBF) and the DFG.

\section{APPENDIX A: INFLUENCE OF CUTOFF FREQUENCY}

In the BCS theory, the electron-phonon coupling is evaluated over an energy range $E_{F} \pm \hbar \omega_{c}$, where $\omega_{c}$ is given by the Debye frequency $\omega_{c}=\omega_{D}$. This is, however, a simplification that might be questionable when being applied to the PE. In order to rule out influences of the cutoff frequency, we plot in Fig. 7 the calculated spectra for the system $80 \mathrm{~nm} \mathrm{Au}$ on $320 \mathrm{~nm} \mathrm{Al}$ at a temperature of $270 \mathrm{mK}$ for four values of the cutoff frequency $\left(\omega_{c}=10 \omega_{D}, \omega_{c}=\omega_{D}, \omega_{c}=0.5 \omega_{D}\right.$, and $\left.\omega_{c}=0.05 \omega_{D}\right)$ for two values of $I P(I P=0$ and $I P=0.6)$.

The inset shows the induced energy gap as a function of $\omega_{c}$ for this thickness. The choice of the cutoff frequency $\omega_{c}$ in Eq. (4) has a small influence within the range $0.5 \omega_{D}<\omega_{c}$. We can rule out that the larger observed gap in Au can be explained by a different effective $\omega_{c, \mathrm{Au}}$.

\section{APPENDIX B: THE SYSTEM Al/Pd}

In materials that are close to ferromagnetism, like palladium spin fluctuations, so-called paramagnons suppress superconductivity. ${ }^{9}$ The influence of the paramagnons can be taken into account by introducing the parameter $\lambda_{s f}$ into the Usadel equation: ${ }^{25}$

$$
\begin{aligned}
\frac{D_{N, S}}{2} \frac{\partial^{2} \theta(x)}{\partial x^{2}}= & -i\left(1+\lambda_{s f}\right) E \sin \theta(x)-\Delta_{N, S}(x) \cos \theta(x) \\
& +\Gamma_{s f} \sin [2 \theta(x)] .
\end{aligned}
$$

So far, no intrinsic superconductivity could be observed in $\mathrm{Pd},{ }^{41}$ despite its strong electron-phonon-coupling. ${ }^{42-44}$ Palladium has a complicated band structure, with $\mathrm{s}$ and $\mathrm{d}$ electrons contributing to the electronic transport. ${ }^{45,46}$ This makes the determination of the diffusion constant based on experimental values of the conductivity more difficult than for the other metals discussed above. Dumoulin et al. ${ }^{47}$ stated that the influence of the d electrons would be dominant for the $\mathrm{PE}^{47}$ and the influence of the paramagnons would be weak. Later, Mitrović ${ }^{48}$ reanalyzed the data, showing that the small 

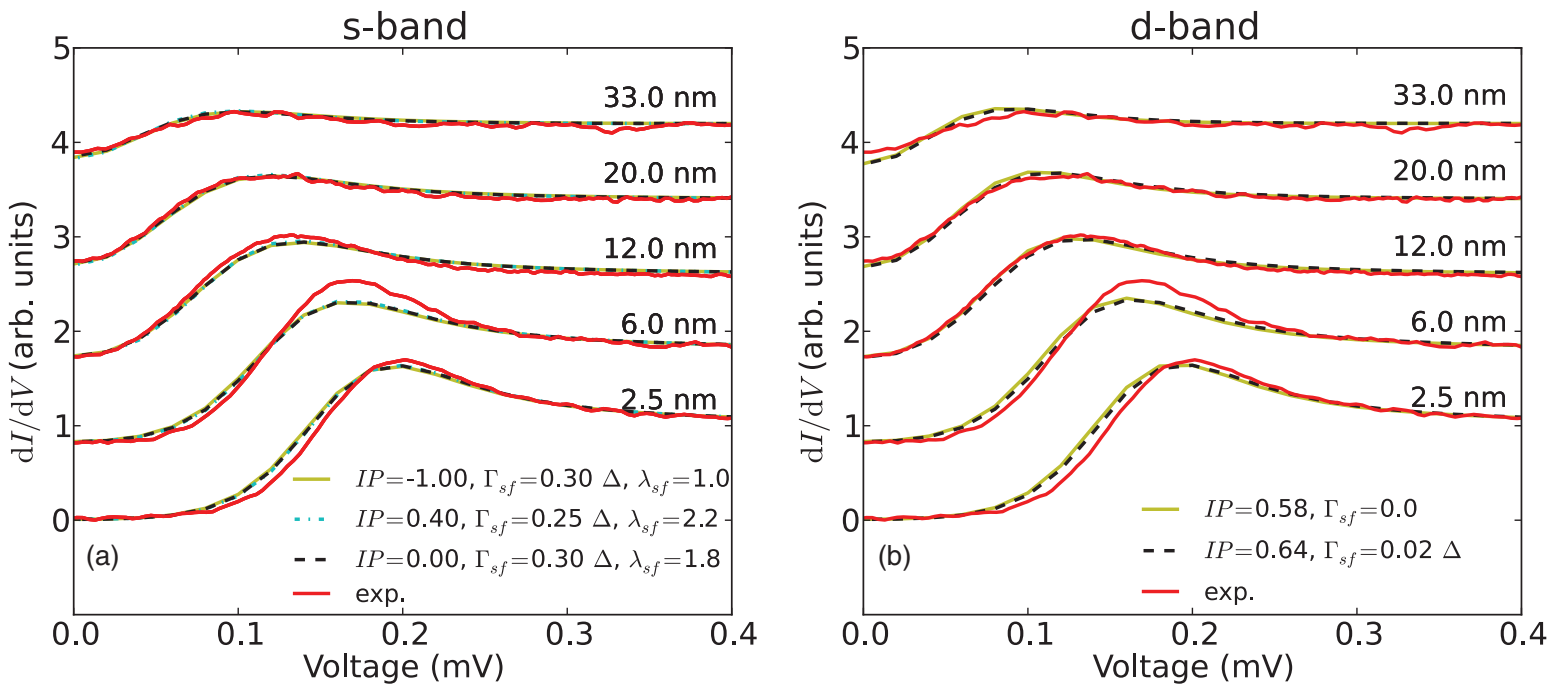

FIG. 8. (Color online) (a) Spectra measured on Al/Pd samples and calculated DOS with the diffusion constant computed from the properties of the s electrons in Pd. (b) Same as (a) but using the d electrons.

influence of the paramagnons might be due to the cancellation effect of a strong electron-phonon contribution and a strong influence of paramagnons. We find a similar situation for our data. Other authors use the band parameters of the s electrons for their calculations of the PE in $\mathrm{Pd} .{ }^{33,35} \mathrm{We}$ can fit our data using the values of $d$ electrons as well as s electrons, but we find different values for $I P$ and for $\lambda_{s f}$ [see Fig. 8]. The second assumption corresponds to a value of the diffusion constant of $D=1.72 \times 10^{-3} \mathrm{~m}^{2} / \mathrm{s}$. Figure 8 (a) shows the result of the fit procedure assuming $I P=0.4, I P=0$, and $I P=-1$. The further fit parameters are $\Gamma_{s f}$ and $\lambda_{s f}$. While the best-fit value for $\Gamma_{s f}=0.3 \Delta$ is very robust, several combinations of $I P$ and $\lambda_{s f}$ result in similar fit quality. Changes in $I P$ can be compensated by a change in $\lambda_{s f}$. The data cannot be described without taking $\lambda_{s f}$ into account. The $\lambda_{s f}$ values are smaller than those deduced by Kontos et al. ${ }^{25}$ from studies of the PE in nanostructured devices of $\mathrm{Nb}$ and $\mathrm{Pd}$. Negative $I P$ values correspond to repulsive interaction and result in a reduction of the minigap, as do the paramagnons described by $\lambda_{s f}$. Negative IP seems thus somewhat redundant to $\lambda_{s f}$, but the influence of the shape of the spectra is slightly different. When using the values of the d electrons for calculating the diffusion constant $D=2.2 \times 10^{-4} \mathrm{~m}^{2} / \mathrm{s}$, the data are well described by a positive value of $I P=0.58$. A slight improvement can be achieved by introducing a spin-flip constant $\Gamma_{s f}=0.02 \Delta$ in combination with $I P=0.64$. Interestingly, no $\lambda_{s f}$ is necessary to describe the spectra when using the d electrons. In that sense, one might conclude like Dumoulin et $a l .{ }^{47}$ that the $\mathrm{d}$ band is the important one for the coupling to the Cooper pairs, because less parameters are needed. We note that we neglected $\gamma_{B}$ throughout this analysis because it would have added yet another parameter. When analyzing data recorded at higher temperatures (not shown), we find a similar broad variation of combinations of the fitting parameters. Summarizing, our results are in agreement with previously reported results, but we are unable to solve the existing ambiguity of the question of which electronic band would be the dominant one for heterodevices with superconductors. Realistically, both bands, which might include differing $I P, \lambda_{s f}$, and $\Gamma_{s f}$ values, will contribute. Obtaining meaningful values for these manifold independent parameters by fitting the experimental spectra is impossible.
*Now at Attocube Systems AG, Königinstraße 11a RGB, 80539 München, Germany.

${ }^{1}$ W. L. McMillan, Phys. Rev. 167, 331 (1968).

${ }^{2}$ R. Bauer, A. Schmid, P. Pavone, and D. Strauch, Phys. Rev. B 57, 11276 (1998).

${ }^{3}$ R. F. Hoyt, H. N. Scholz, and D. O. Edwards, Phys. Lett. A 84, 145 (1981).

${ }^{4}$ C. Buchal, R. M. Mueller, F. Pobell, M. Kubota, and H. R. Folle, Solid State Commun. 42, 43 (1982).

${ }^{5}$ R. A. Buhrman and W. P. Halperin, J. Low Temp. Phys. 16, 409 (1974).

${ }^{6}$ H. Bary-Soroker, O. Entin-Wohlman, and Y. Imry, Phys. Rev. Lett. 101, 057001 (2008).
${ }^{7}$ N. Nishida, M. Yamaguchi, T. Furubayashi, K. Morigaki, H. Ishimoto, and K. Ono, Solid State Commun. 44, 305 (1982).

${ }^{8}$ B. Sundqvist, J. Neve, and O. Rapp, Phys. Rev. B 32, 2200 (1985).

${ }^{9}$ N. F. Berk and J. R. Schrieffer, Phys. Rev. Lett. 17, 433 (1966).

${ }^{10}$ P. G. de Gennes and D. Saint-James, Phys. Lett. 4, 151 (1963).

${ }^{11}$ S. Guéron, H. Pothier, N. O. Birge, D. Esteve, and M. H. Devoret, Phys. Rev. Lett. 77, 3025 (1996).

${ }^{12}$ N. Moussy, H. Courtois, and B. Pannetier, Europhys. Lett. 55, 861 (2001).

${ }^{13}$ Y. Levi, O. Millo, N. D. Rizzo, D. E. Prober, and L. R. Motowidlo, Phys. Rev. B 58, 15128 (1998).

${ }^{14}$ A. D. Truscott, R. C. Dynes, and L. F. Schneemeyer, Phys. Rev. Lett. 83, 1014 (1999). 
${ }^{15}$ M. Vinet, C. Chapelier, and F. Lefloch, Phys. Rev. B 63, 165420 (2001).

${ }^{16}$ S. H. Tessmer, M. B. Tarlie, D. J. VanHarlingen, D. L. Maslov, and P. M. Goldbart, Phys. Rev. Lett. 77, 924 (1996).

${ }^{17}$ W. Belzig, F. K. Wilhelm, C. Bruder, G. Schön, and A. D. Zaikin, Superlattices Microstruct. 25, 1251 (1999).

${ }^{18}$ W. Belzig, C. Bruder, and G. Schön, Phys. Rev. B 54, 9443 (1996).

${ }^{19}$ K. D. Usadel, Phys. Rev. Lett. 25, 507 (1970).

${ }^{20}$ S. H. Tessmer, D. J. Van Harlingen, and J. W. Lyding, Phys. Rev. Lett. 70, 3135 (1993).

${ }^{21}$ R. J. Soulen et al., Science 282, 85 (1998).

${ }^{22}$ F. Pérez-Willard, J. C. Cuevas, C. Sürgers, P. Pfundstein, J. Kopu, M. Eschrig, and H. v. Löhneysen, Phys. Rev. B 69, 140502 (2004).

${ }^{23}$ H. le Sueur, P. Joyez, H. Pothier, C. Urbina, and D. Esteve, Phys. Rev. Lett. 100, 197002 (2008).

${ }^{24}$ J. Bardeen, L. N. Cooper, and J. R. Schrieffer, Phys. Rev. 108, 1175 (1957).

${ }^{25}$ T. Kontos, M. Aprili, J. Lesueur, X. Grison, and L. Dumoulin, Phys. Rev. Lett. 93, 137001 (2004).

${ }^{26} \mathrm{G}$. Grimvall, The Electron-Phonon Interaction in Metals (NorthHolland, Amsterdam, 1981), Vol. 16.

${ }^{27}$ C. Debuschewitz, F. Münstermann, V. Kunej, and E. Scheer, J. Low Temp. Phys. 147, 525 (2007).

${ }^{28}$ M. Khoshnevisan, W. P. Pratt, P. A. Schroeder, S. Steenwyk, and C. Uher, J. Phys. F 9, L1 (1979).

${ }^{29}$ W. Belzig, Ph.D. thesis, University of Karlsruhe, 1999.

${ }^{30}$ A. Tesauro, A. Aurigemma, C. Cirillo, S. L. Prischepa, M. Salvato, and C. Attanasio, Supercond. Sci. Technol. 18, 1 (2005).

${ }^{31}$ A. V. Zaitsev, Sov. Phys. JETP 59, 1015 (1984).

${ }^{32}$ M. Y. Kupriyanov and V. F. Lukichev, Sov. Phys. JETP 67, 1163 (1988).

${ }^{33}$ T. S. Khaire, W. P. Pratt, and N. O. Birge, Phys. Rev. B 79, 094523 (2009).
${ }^{34}$ S. Pilgram, W. Belzig, and C. Bruder, Phys. Rev. B 62, 12462 (2000).

${ }^{35}$ A. Potenza, M. S. Gabureac, and C. H. Marrows, Phys. Rev. B 76, 014534 (2007).

${ }^{36}$ A. K. Gupta, L. Cretinon, N. Moussy, B. Pannetier, and H. Courtois, Phys. Rev. B 69, 104514 (2004).

${ }^{37}$ R. F. Hoyt and A. C. Mota, Solid State Commun. 18, 139 (1976).

${ }^{38}$ K. E. Gray, Phys. Rev. Lett. 28, 959 (1972).

${ }^{39}$ W. L. McMillan, Phys. Rev. 175, 537 (1968).

${ }^{40}$ E. Scheer, W. Belzig, Y. Naveh, M. H. Devoret, D. Esteve, and C. Urbina, Phys. Rev. Lett. 86, 284 (2001).

${ }^{41}$ R. A. Webb, J. B. Ketterson, W. P. Halperin, J. J. Vuillemin, and N. B. Sandesara, J. Low Temp. Phys. 32, 659 (1978).

${ }^{42}$ F. J. Pinski, P. B. Allen, and W. H. Butler, Phys. Rev. Lett. 41, 431 (1978).

${ }^{43}$ T. Takezawa, H. Nagara, and N. Suzuki, Phys. Rev. B 71, 012515 (2005).

${ }^{44}$ S. K. Bose, J. Phys. Condens. Matter 21, 025602 (2009).

${ }^{45}$ F. J. Pinski, P. B. Allen, and W. H. Butler, Phys. Rev. B 23, 5080 (1981).

${ }^{46}$ I. I. Mazin, E. Savitskii, and Y. A. Uspenskii, J. Phys. F 14, 167 (1984).

${ }^{47}$ L. Dumoulin, P. Nedellec, and P. M. Chaikin, Phys. Rev. Lett. 47, 208 (1981).

${ }^{48}$ B. Mitrović, Solid state commun. 47, 51 (1983).

${ }^{49} \mathrm{The}$ roughness of the $\mathrm{Al}$ films is less than a 2-nm root mean square; the roughness of the Au film on both layer thicknesses of $\mathrm{Al}$ is equal to and increases with the absolute thickness of the Au film. It is roughly $15 \mathrm{~nm}$ for the thickest layers. We therefore took spectra on several positions of the sample and did not find significant variation of the spectra from position to position. 